\title{
Detección de Aedes albopictus (Skuse) (Diptera: Culicidae) en la ciudad de Cali, Valle del Cauca, Colombia
}

\author{
María Elena Cuéllar-Jiménez ${ }^{1}$, Olga Lucía Velásquez-Escobar ${ }^{2}$, Ranulfo González-Obando ${ }^{3}$, \\ Carlos Andrés Morales-Reichmann ${ }^{4}$ \\ 1 Unidad de Entomología, Laboratorio de Salud Pública Departamental del Valle del Cauca, Cali, Colombia \\ 2 Programa de Enfermedades Transmitidas por Vectores, Unidad Ejecutora de Saneamiento del Valle del \\ Cauca, Cali, Colombia \\ ${ }^{3}$ Sección de Entomología, Departamento de Biología, Facultad de Ciencias, Universidad del Valle, Cali, \\ Colombia \\ ${ }^{4}$ Grupo de Epidemiología y Salud Pública, Área de Salud Ambiental, Programa Dengue, Secretaría de Salud \\ Pública Municipal de Cali, Cali, Colombia
}

Introducción. Aedes albopictus es el segundo vector más importante del virus del dengue en el sudeste asiático después de Aedes aegypti. Su ingreso a las Américas ocurrió en 1985; los estudios realizados demuestran su potencial como vector en este continente. En Colombia, la especie se ha reportado en Leticia (Amazonas) en 1998 y en Buenaventura (Valle del Cauca) en 2001. Los últimos hallazgos indican que este insecto continúa avanzando hacia el interior del país.

Objetivo. Informar sobre la presencia de A. albopictus en el municipio de Cali, Valle del Cauca, Colombia.

Materiales y métodos. Desde el año 2002, semanalmente se realizan muestreos en larvitrampas de 17 estaciones del departamento. La determinación de la especie Ae. albopictus encontrada en Cali, fue realizada en la Unidad de Entomología del Laboratorio de Salud Pública Departamental, diagnóstico que fue confirmado en los Laboratorios de Entomología de la Universidad del Valle y del Instituto Nacional de Salud en Bogotá.

Resultados. Durante abril a junio de 2006, se encontraron larvas de $A$. albopictus en seis estaciones de muestreo ubicadas entre el noroccidente y nororiente de Cali, una de ellas en el área periurbana del municipio de Yumbo.

Conclusión. Es importante integrar el control de $A$. aegypti y $A$. albopictus en un solo programa. Se recomienda intensificar la vigilancia en los municipios y departamentos vecinos con el fin de limitar a tiempo el avance de la especie.

Palabras clave: Aedes, vectores de enfermedades, dengue, vigilancia epidemiológica, Colombia.

Detection of Aedes albopictus (Skuse) (Diptera: Culicidae) in the city of Cali, Valle del Cauca, Colombia

Introduction. Aedes albopictus is the second most important dengue virus vector in the Asian southeast after Aedes aegypti. Its entrance into the Americas occurred in 1985, and laboratory studies performed show its potential as a vector in this continent as well. In Colombia, this specie has been reported in Leticia (Amazonas) in 1998 and Buenaventura (Valle del Cauca) in 2001. The latest discoveries show that this mosquito continues to advance toward the country's interior.

Objective. To inform the presence of $A$. albopictus is documented in the city of Cali, Valle del Cauca, Colombia.

Materials and Methods. Since 2002, weekly sampling has been performed using larval traps located at seventeen stations. The identification of the $A$. albopictus species, was carried out in the Unidad de Entomología, Laboratorio de Salud Pública Departamental. These identifications were confirmed in the Entomology Laboratory at Universidad del Valle and the National Institute of Health in Bogotá. 
Results. From April to June of 2006, larvae of $A$. albopictus were found in six sampling stations located between northwest and northeast of Cali, one of them in the suburban area of the Yumbo city.

Conclusion. The control of $A$. aegypti and $A$. albopictus must be integrated into a single program. The surveillance in the cities and nearest departments must be intensified with the objective of limiting the advancement of $A$. albopictus.

Key words: Aedes, disease vectors, dengue, epidemiologic surveillance, Colombia.

Aedes albopictus (Skuse), un mosquito originario del sudeste asiático, es el segundo vector más importante del virus del dengue en este continente después de Aedes aegypti (Linnaeus) (1). En las Américas fue reportado en Texas, Estados Unidos en 1985 (2) y meses más tarde en Río de Janeiro, Brasil (3). Posteriormente, siete años después su distribución se extendió a países de Centro y Suramérica como República Dominicana y México (4). En 1995 fue reportado en Bolivia (4), (un solo ejemplar) Molinedo (comunicación personal), donde fue erradicado, Cuba (5) y Honduras (6,7). En 1995 se encontró en Guatemala (8) y en 1996 en El Salvador (4). En 1997 fue hallado en Islas Caimán (9), en 1998 en Argentina (10), en 1999 en Paraguay (Cousiño, comunicación personal), en 2002 en Panamá (11) y en 2003 en Uruguay (12) y Nicaragua (13) (figura 1).

En Colombia el primer reporte de esta especie se efectuó en el municipio de Leticia, Amazonas, en 1998 donde no hay presencia de $A$. aegypti $(14,15)$. Posteriormente, en el 2001 A. albopictus fue detectado en el municipio de Buenaventura, Valle del Cauca (16); tres años más tarde fue encontrado en Loboguerrero, corregimiento del área rural del municipio de Dagua, Valle del Cauca (Suárez M, datos sin publicar). Desde entonces, se ha estado registrando periódicamente en esta localidad.

Se cree que la introducción y el establecimiento de $A$. albopictus en el continente americano se debe principalmente al comercio de materiales transportados por vía aérea y marítima sin

Correspondencia:

María Elena Cuéllar-Jiménez, Laboratorio de Salud Pública Departamental del Valle del Cauca, Carrera 76 No. 4-30, Barrio Nápoles, Santiago de Cali.

mariaelenacuellar@graffiti.net

Recibido: 19/09/06; aceptado: 22/02/07 programas de vigilancia entomológica adecuados en la mayoría de los países; así como también, a las condiciones ambientales existentes que favorecen la reproducción del insecto (17). Específicamente, se ha comprobado que la introducción de la especie a los Estados Unidos se produjo en neumáticos transportados en grandes contenedores de carga provenientes del Japón y se sospecha que la importación al Brasil se efectuó en tocones de bambú provenientes del sudeste de Asia (18).

La importancia epidemiológica de este insecto en el continente asiático se debe a la transmisión del virus dengue, principalmente en áreas rurales (1), el cual puede ser transmitido por vía transovarial y sexual $(19,20)$. Pero, además, porque es vector potencial del virus Chikungunya

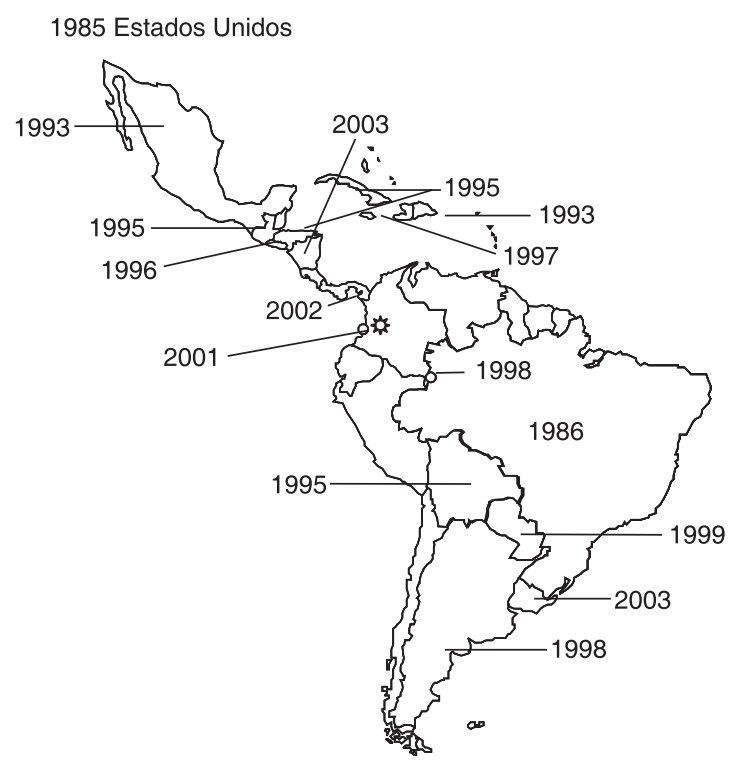

Figura 1. Distribución de Aedes albopictus en las Américas 1985-2006 (elaboró C. A. Morales). 
(21), del virus de la encefalitis japonesa (22) y de larvas de Dirofilaria immitis (23).

Estudios realizados en campo y laboratorio indican que $A$. albopictus es susceptible a la infección por agentes patógenos de importancia médica. Bajo condiciones de laboratorio es un competente vector de 22 arbovirus, incluyendo aquéllos de importancia en salud pública (24).

Poco se ha demostrado sobre el papel de $A$. albopictus en la transmisión de enfermedades de importancia en salud pública en las Américas y, en algunos casos, se ha aseverado que no está involucrado como vector en el campo de las enfermedades $(25,26)$. Sin embargo, en 1992 se observaron ejemplares de esta especie infectados naturalmente con el virus de la encefalitis del este en Florida (27). Así mismo, en 1993 en el municipio de Campos Altos, estado de Minas Gerais (Brasil), se confirmaron tres casos de dengue en un área no infestada por $A$. aegypti. En este sitio, se encontraron larvas de $A$. albopictus infectadas con virus dengue serotipo 1 (28). Dos años después, en 1995, se reportó la infección natural de larvas con virus dengue en la ciudad de Reynosa, Tamaulipas (México), encontrando también machos infectados, lo que comprueba la transmisión transovárica del virus en campo (29). Sumado a lo anterior, en agosto del 2002, se confirmó por medio de los Centers for Disease Control and Prevention de Estados Unidos la infección de $A$. albopictus con el virus del oeste del Nilo (30). Finalmente, en Buenaventura entre los años de 2002 y 2004, se encontraron ejemplares infectados con virus dengue de los serotipos 1 y 2 (31).

Por otro lado, en áreas donde cohabitan $A$. aegypti y $A$. albopictus, este último puede potencialmente servir como vector de mantenimiento de los serotipos del virus dengue en zonas endémicas, con la posibilidad de que aparezcan nuevos focos por transmisión vertical (32-34).

Teniendo en cuenta la importancia potencial de A. albopictus como vector de arbovirus en Colombia, el objetivo de este informe es dar a conocer la presencia de la especie en el área urbana del municipio de Cali, Valle del Cauca, Colombia.

\section{Materiales y métodos}

Después de detectarse la presencia de $A$. albopictus en el Valle del Cauca, en enero de 2002 se inició un programa de vigilancia de la especie en todo el departamento. Las actividades se realizan en coordinación con la Unidad Ejecutora de Saneamiento del Valle del Cauca, la Secretaría de Salud Pública Municipal de Cali y la Unidad de Entomología del Laboratorio de Salud Pública Departamental. Este programa consiste en la instalación de larvitrampas en zonas portuarias de concentración humana, o cerca de viviendas, de alto riesgo por el transporte y descargue de mercancías y pasajeros, de acuerdo con las recomendaciones de la Organización Panamericana de la Salud (OPS) y de la Organización Mundial de la Salud (OMS) (18). Además, en estas zonas confluyen los puntos estratégicos de monitoreo que se encuentran ubicados en el aeropuerto Alfonso Bonilla Aragón de la ciudad de Palmira, puertos secos de Guadalajara de Buga, Yotoco, área periurbana de Yumbo y Dagua; el corregimiento de Loboguerrero, municipio de Dagua y Santiago de Cali con seis estaciones de vigilancia para esta especie. En cada estación se tienen colocadas un total de cuatro larvitrampas. Las estaciones de Cali se encuentran ubicadas entre el noroeste y el noreste de la ciudad.

La larvitrampa consiste en una llanta mediana de vehículo, partida a la mitad y amarrada por sus extremos con un alambre formando un aro, del cual es colgada a $30 \mathrm{~cm}$ de altura sobre el nivel del piso. Se ubicaron en sitios protegidos de la luz directa del sol y de las aguas lluvias y en su interior se vertieron, aproximadamente, dos litros de agua de grifo, con el propósito de servir como sitios de postura (35). Las larvitrampas se inspeccionaron semanalmente, realizando cinco tomas de cucharón colmado ( $\approx 250 \mathrm{ml}$ ) en cada una. Si presentaban larvas se tomaba una muestra por cada larvitrampa positiva. Para esto, se recolectaban de cuatro a ocho ejemplares en un vial con alcohol al $70 \%$. Por lo tanto, el número de muestras en cada estación dependió del número de larvitrampas positivas para Aedes spp. Las muestras debidamente rotuladas se remitieron mensualmente a la Unidad de Entomología del Laboratorio de Salud Pública Departamental. 
Desde el 2002, cuando se inició el programa de vigilancia de la especie, hasta junio de 2006, el total de muestras analizadas fue de 1.619.

Cuando se detectó la especie, se realizó una búsqueda activa en cada una de las tres estaciones de muestreo donde se encontró inicialmente, con el objetivo de capturar más ejemplares. Para llevar a cabo lo anterior, se inspeccionó en los alrededores de las estaciones positivas, buscando depósitos de agua dentro de viviendas, talleres y montallantas, y se inspeccionaron sumideros y otros posibles criaderos. Cuando los criaderos fueron positivos, se recuperaron todas las larvas de Aedes y se realizó la cuantificación por especie.

El hallazgo de $A$. albopictus en Cali ocurrió por primera vez en abril de 2006, y se siguió detectando en mayo y julio. La determinación de las larvas y adultos se realizó utilizando la clave de Darsie (36). La confirmación fue realizada en la Sección de Entomología de la Universidad del Valle (Cali) y en el Laboratorio de Entomología del Instituto Nacional de Salud de Bogotá.

\section{Resultados}

De las muestras obtenidas en larvitrampas ubicadas en seis estaciones de muestreo de la Cali y una en el área periurbana del municipio de Yumbo, seis resultaron positivas a $A$. albopictus durante los meses de abril a junio de 2006. Además, en cada estación sólo se encontró una larvitrampa positiva para esta especie. En abril, las estaciones donde apareció por primera vez la especie fueron la terminal de transportes y las bodegas de Almaviva y Aloccidente de la ciudad de Cali, ubicadas al norte la primera y al noreste las otras dos. Para el mes de mayo, apareció nuevamente en la estación de Almaviva y se detectó por primera vez en la estación de La Balastrera, ubicada al noroccidente de la ciudad. En junio, la especie fue detectada en dos estaciones nuevas, el Retén Forestal (noroccidente de la ciudad, carretera Cali-Buenaventura) y las bodegas de Alpopular (área periurbana del municipio de Yumbo). En todas las estaciones, las larvas de $A$. albopictus se encontraron asociadas a la especie $A$. aegypti (cuadro 1 ). Con excepción de la muestra tomada en el Retén Forestal en el mes de junio, la proporción de larvas de $A$. aegypti superó la de $A$. albopictus.

Como resultado de la búsqueda de criaderos alrededor de las larvitrampas positivas, en la terminal de transporte intermunicipal de la ciudad se encontraron dos llantas positivas para $A$. albopictus y $A$. aegypti. De la densidad total de larvas de Aedes ( $\mathrm{N}=137)$, encontrada en las dos llantas, $10,2 \%$ correspondió a $A$. albopictus, 8,1\% para la del Parqueadero Central y $12,0 \%$ para la del prado occidental (cuadro 2). A partir de las pupas y de una de las larvas recolectadas, se obtuvieron cinco adultos, cuatro de la especie $A$. aegypti y uno de $A$. albopictus.

\section{Discusión}

Después del hallazgo de $A$. albopictus en Cali, los resultados indican que aún no es la especie dominante en los criaderos donde cohabita con A. aegypti. Sin embargo, es curioso que después

Cuadro 1. Proporción de larvas de Aedes aegypti/A. albopictus recolectadas en larvitrampas de seis estaciones de muestreo en el área urbana de Cali, entre abril y junio de 2006

\begin{tabular}{|c|c|c|c|}
\hline Mes & Estación de muestreo* & $\begin{array}{l}\text { Número de larvas } \\
\text { por muestra }\end{array}$ & $\begin{array}{c}\text { Proporción A. aegypti/ } \\
\text { A. albopictus }\end{array}$ \\
\hline \multirow[t]{3}{*}{ Abril } & Terminal de transportes & 6 & 2,0 \\
\hline & Almaviva & 7 & 2,5 \\
\hline & Al occidente & 5 & 4,0 \\
\hline \multirow[t]{2}{*}{ Mayo } & Almaviva & 8 & 7,0 \\
\hline & La Balastrera & 4 & 3,0 \\
\hline \multirow[t]{2}{*}{ Junio } & Retén forestal & 6 & 0,2 \\
\hline & Alpopular** & 5 & 1,5 \\
\hline
\end{tabular}

* De cuatro larvitrampas instaladas en cada estación, siempre se encontró una sola larvitrampa positiva para A. albopictus durante los meses de muestreo.

${ }^{* *}$ Area periurbana del municipio de Yumbo 
Cuadro 2. Relación de la densidad de larvas de Aedes aegypti y $A$. albopictus y pupas recolectadas en dos llantas encontradas en la terminal de transportes de la ciudad de Cali.

\begin{tabular}{|c|c|c|c|c|c|}
\hline \multirow{3}{*}{$\begin{array}{l}\text { Ubicación de } \\
\text { las Ilantas }\end{array}$} & \multicolumn{4}{|c|}{ No. de larvas por especie } & \multirow{3}{*}{$\begin{array}{l}\text { No. de } \\
\text { Pupas }\end{array}$} \\
\hline & \multicolumn{2}{|c|}{ A. aegypti } & \multicolumn{2}{|c|}{ A. albopictus } & \\
\hline & $\mathbf{N}$ & $\%$ & $\mathbf{N}$ & $\%$ & \\
\hline Parqueadero central & 57 & 91,9 & 5 & 8,1 & 4 \\
\hline Prado área occidental & 66 & 88,0 & 9 & 12,0 & 0 \\
\hline Total & 123 & 89,8 & 14 & 10,2 & 4 \\
\hline
\end{tabular}

de cinco años de vigilancia continua, se haya detectado en una misma fecha (abril) en sitios distantes; y en los dos meses siguientes en estaciones ubicadas en sitios completamente opuestos, incluso en el área periurbana de Yumbo. Estos hechos podrían indicar que posiblemente la especie llegó mucho antes de ser detectada en las larvitrampas de las estaciones de muestreo. Una de las suposiciones que permitirían entender los hechos, es que este tipo de trampa sólo detecta la especie a partir de ciertas densidades. La segunda es que, una vez ingresó, fue favorecida por las condiciones ambientales que prevalecieron durante la estación lluviosa de comienzos de año, $y$, por lo tanto, sus poblaciones proliferaron hasta alcanzar diferentes puntos de la ciudad.

Sobre su origen y modo de ingreso a Cali, no existe información alguna. Sin embargo, se sospecha que la población encontrada en Cali procede del municipio de Buenaventura donde se estima que lleva más de cinco años después de su establecimiento (16). También existe la posibilidad que la población no tenga su origen en el puerto de dicho municipio, sino que provenga de otro país a partir del cual se transportaron pasivamente en contenedores con mercancías (posiblemente llantas) hasta llegar a la ciudad de Cali y entraron por el terminal marítimo. El mercado de llantas reencauchadas a través de los puertos de Colombia es bastante elevado; entre 1989 y 1994 ingresaron a Colombia 1'374.314 llantas usadas provenientes de Estados unidos y 474 provenientes de Japón (9).

Quizá el principal interrogante que existe es saber cuál es la importancia epidemiológica actual de esta especie en el Valle del Cauca y en otros departamentos del país donde pueda colonizar.
La mayor preocupación tiene que ver con su competencia vectorial en la transmisión de los virus del dengue, fiebre amarilla y del virus del oeste del Nilo de la cepa colonizadora en condiciones experimentales.

Hasta ahora $A$. aegypti ha sido el único vector que interviene en la transmisión urbana de dengue y fiebre amarilla en las Américas (1). Sin embargo, se cree que una de las razones por la que no ha ocurrido la urbanización de la fiebre amarilla ha sido la ausencia de un vector que pueda utilizar eficazmente los medios urbano y suburbano y las zonas rurales o selváticas. Puesto que $A$. albopictus puede proliferar en las zonas urbanas, rurales y selváticas asociado a la actividad humana, puede servir de puente entre los ciclos selvático y urbano de la fiebre amarilla. En el laboratorio, $A$. albopictus se ha infectado por vía oral con el agente causante de la fiebre amarilla y se ha demostrado que transmite este virus a los monos (18). Por otro lado, es igualmente preocupante el papel que pueda jugar en la dispersión del virus del Nilo occidental ante la eventual llegada en aves migratorias.

Se sabe que el problema de $A$. albopictus no es muy distinto del causado por $A$. aegypti en el sudeste de Asia, ya que estos dos mosquitos tienen similitudes en lo que respecta a la transmisión de la enfermedad, criaderos, etc. (24). En cuanto a los sitios de cría en Colombia, después de la detección de $A$. albopictus en Buenaventura, se realizó un estudio en el área urbana y suburbana de este municipio, en el cual se encontró que $9,6 \%$ de las viviendas tenían larvas de $A$. albopictus, $21,2 \%$ de $A$. aegypti y $7,1 \%$ de ambas especies. Además, los criaderos más frecuentemente encontrados con $A$. albopictus fueron los categorizados como "diversos", mientras que en tanques bajos se encontró asociación entre $A$. albopictus y $A$. aegypti (Suárez $\mathrm{M}$, datos sin publicar). En Leticia (Colombia), donde se realizó un estudio sobre los criaderos de $A$. albopictus, éstos fueron encontrados en el peridomicilio de las viviendas y en su mayoría fueron depósitos inservibles temporales, recién establecidos y con exposición parcial al sol o en la sombra (Carvajal JJ, Moncada LI, Rodríguez H, Pérez LP, Olano VA). Caracterización de los criaderos de larvas 
de Aedes (Stegomyia) albopictus (Diptera: Culicidae) en el municipio de Leticia (Amazonas, Colombia). Resúmenes XXXI Congreso SOCOLEN. Bogotá; 1994. p.45).

En Cali, hasta el momento se ha efectuado búsqueda de larvas en los sitios positivos en larvitrampas. Se han inspeccionado 198 viviendas y 932 depósitos, encontrándose un solo ejemplar dentro de las viviendas. En el futuro se ampliará la vigilancia instalando larvitrampas en cada comuna y zona rural (33 sitios), para detectar su distribución y factibilidad de control.

En Cali, al igual que en el municipio de Buenaventura y otros sitios donde coexisten las dos especies, es necesario ampliar los estudios para determinar además de los tipos de criaderos más frecuentes, cuáles son los que producen mayor cantidad de adultos, para valorar la posibilidad de establecer un programa de control para $A$. albopictus e incorporarlo dentro del programa existente para $A$. aegypti.

Muchas de las recomendaciones aprobadas por la OPS para el manejo de $A$. aegypti pueden ser aplicables a $A$. albopictus en el Valle del Cauca (18). No obstante, para poder adoptar medidas adecuadas de control, es importante, conocer la distribución alcanzada por $A$. albopictus en Cali (zona urbana y rural), realizar estudios sobre la tipificación de criaderos de la especie y levantar índices para determinar la densidad de individuos (larvas y adultos) a lo largo del año. Finalmente, es importante efectuar esfuerzos para limitar la proliferación de la especie y, por tanto, su rango de dispersión hacia otras localidades. Para los municipios y departamentos vecinos se recomienda intensificar la vigilancia ubicando larvitrampas en sitios estratégicos y hábitats preferidos del vector.

Con este reporte se amplia la distribución de $A$. albopictus en el país y más concretamente en el departamento del Valle del Cauca y se reitera el riesgo epidemiológico que significa por su gran potencial como vector.

\section{Agradecimientos}

A Helena Brochero y Betsy Bello del Instituto Nacional de Salud, por confirmar la determinación de la especie y por sus oportunas sugerencias.
A Irne Arana, auxiliar de ETV de la Unidad Ejecutora de Saneamiento del Valle del Cauca, asignado a la Secretaría de Salud Municipal de Cali y encargado de realizar la vigilancia de Aedes albopictus en la ciudad de Cali.

A Pedro Puerta y Edinson Cuéllar, auxiliares de ETV de la Unidad Ejecutora de Saneamiento del Valle del Cauca, por colaborar en la búsqueda intensiva de la especie después de su hallazgo.

\section{Conflicto de intereses}

Los autores manifestamos que no existe ningún conflicto de intereses en torno a los resultados presentados.

\section{Financiación}

Este trabajo fue financiado por la Gobernación del Valle del Cauca, Secretaría de Salud Departamental, Unidad Ejecutora de Saneamiento del Valle del Cauca, Secretaría de Salud Municipal de Cali y contó con el apoyo institucional del Laboratorio de Salud Pública Departamental del Valle del Cauca y la Universidad del Valle.

\section{Referencias}

1. Hawley WA. The biology of Aedes albopictus. J Am Mosq Control Assoc. 1988;4(Suppl.1):1-39.

2. Sprenger D, Wuithiranyagool T. The discovery and distribution of Aedes albopictus in Harris County, Texas. J Am Mosq Control Assoc. 1986;2:217-9.

3. Forattini OP. Identificão de Aedes (Stegomyia) albopictus no Brasil. Rev Saúde Pública. 1986; 20: 244-5.

4. PAHO. The feasibility of eradicating Aedes aegypti in the Americas. Rev Panam Salud Pública. 1997;1:6872.

5. Broche RG, Borja EM. Aedes albopictus in Cuba. J Am Mosq Control Assoc. 1999;15:569-70.

6. Woodall J. Aedes albopictus - Honduras. December 14, 1995. [Consultado: 25 de mayo de 2006]. Disponible en:http://www.promedmail.org/pls/promed/f? $p=2400$ : 1202:14073264455859956808::NO::F2400_P1202_ CHECK_DISPLAY,F2400_P1202_PUB_MAIL_ID:X, 12033.

7. Mejía L. Puerto Cortés en alerta por presencia de "tigre asiático". [Consultado: 25 de mayo 2006 ] Disponible en:http://www.laprensahn.com/valle_nota. php?id0 $4962=11044 \& \mathrm{t}=1148536800$.

8. Ogata K, López-Samayoa A. Discovery of Aedes albopictus in Guatemala. J Am Mosq Control Assoc. 1996;12:503-6. 
9. Reiter P. Aedes albopictus and the world trade in used tires. 1988-1995: the shape of things to come? J Am Mosq Control Assoc. 1998;14:83-94.

10. Schweigmann N, Vezzani D, Orellano P, Kuruc J, Boffi R. Aedes albopictus in an area of Misiones, Argentina. Rev Saúde Pública. 2004;38:136-8.

11. International Society for Infectious Diseases. Panama detects new dengue carrying mosquito. November 8, 2002. [Consultado: 25 de mayo 2006]. Disponible en: http://www.promedmail. org/pls/promed/ $f ? p=2400: 1202: 27859627393929$ 36736::NO::F240 0 P1202_CHECK_DISPLAY,F2400_P1202_PUB MAIL_ID:X,19765.

12. Salvatella R, Rosa R. Culícidos y salud humana en el Uruguay. Entomol Vect. 2003;10:431-6.

13. Lugo EC, Moreno G, Sacaría MA, López MM, López JD, Delgado MA, et al. Scientific Note: Identification of Aedes albopictus in urban Nicaragua. J Am Mosq Control Assoc. 2005;21:325-7.

14. Olano V, Brochero H, Arévalo C, Pérez L, Suárez M, Arroyo J. Evaluación entomológica sobre la presencia de Aedes albopictus en Leticia (Amazonas). Inf Quinc Epidemiol Nac. 1998;3:165-8.

15. Vélez ID, Quiñónez M, Suárez M, Olano V, Murcia LM, Correa E, et al. Presencia de Aedes albopictus en Leticia, Amazonas, Colombia. Biomédica. 1998; 18:192-8.

16. Suárez M. Aedes albopictus (Skuse) (Diptera, Culicidae) en Buenaventura, Colombia. Inf Quinc Epidemiol Nac. 2001;6:221-4.

17. Organización Panamericana de la Salud. Aedes albopictus en las Américas. Reseñas. Bol Of Sanit Panam. 1987;102:624-33.

18. OPS. Aedes albopictus en las Américas. 99a Reunión del Comité Ejecutivo del Consejo Directivo de la OPS. Washington, D.C. Organización Panamericana de la Salud; 1987.

19. Rosen L. Sexual transmission of dengue viruses by Aedes albopictus. Am J Trop Med Hyg. 1987;37:398402.

20. Rosen L, Shroyer DA, Tesh RB, Freier JE, Lien JC. Transovarial transmission of dengue viruses by mosquitoes Aedes albopictus and Aedes aegypti. Am J Trop Med Hyg. 1983;32:1108-19.

21. Mangiafico JA. Chikungunya virus infection and transmission in five species of mosquito. Am J Trop Med Hyg. 1971;20:642-5.

22. Rosen L, Tesh R, Lien JC, Cross JH. Transovarial transmission of Japanese encephalitis virus by mosquitoes. Science. 1978;199:909-11.

23. Chellapha WT, Chellapha GR Jr. Susceptibility of four common Singapore mosquitoes to Dirofilaria immitis Leidy. J Med Entomol. 1968;5:358-61.
24. Estrada-Franco J, Craig G Jr. Biología, relaciones con enfermedades y control de Aedes albopictus (Cuaderno técnico No. 42). Washington D. C.: Organización Panamericana de la Salud; 1995. p.51.

25. Moore CG, Mitchell, CJ. Aedes albopictus in the United States: Ten-year presence and public health implications. Emerg Infect Dis. 1997;3:329-34.

26. Degallier N, Teixeira JM, Soares Sd Sda S, Pereira RD, Pinto SC, Chaib Adade AJ, et al. Aedes albopictus may not be vector of dengue virus in human epidemics in Brazil. Rev Saúde Pública. 2003;37:386-7.

27. Mitchell CJ, Haramis LD, Karabatsos N, Smith GC, Starwalt VJ. Isolation of La Crosse, Cache Valley, and Potosi viruses from Aedes mosquitoes (Diptera: Culicidae) collected at used-tire sites in Illinois during 19941995. J Med Entomol. 1998;35:573-7.

28. Serufo JC, Oca HM, Tavares VA, Souza AM, Rosa RV, Jamal MC, et al. Isolation of dengue virus type 1 from larvae of Aedes albopictus in Campos Altos City, State of Minas Gerais, Brazil. Mem Inst Oswaldo Cruz. 1993;88:503-4.

29. Ibañez-Bernal S, Briceño B, Mutebi SP, Argot E, Rodríguez G, Martínez-Campos C et al. First record in America of Aedes albopictus naturally infected with dengue virus during the 1995 outbreak at Reynosa, México. Med Vet Entomol. 1997;11:305-9.

30. Holick J, Kile A, Ferraro W, Delaney RR, Iwaseczko M. Discovery of Aedes albopictus infected with west nile virus in Southeastern Pennsylvania. J Am Mosq Control Assoc. 2002;18:131.

31. Méndez F, Barreto M, Arias JF, Rengifo G, Muñoz J, Burbano ME, et al. Human and mosquito infections by dengue viruses during and after epidemics in a dengue-endemic region of Colombia. Am J Trop Med Hyg 2006;74:678-83.

32. Gubler DJ. Dengue. En: Monath TP, editor. The arboviruses: epidemiology and ecology. Vol II. Boca Ratón: CRC Press; 1988.p.223-80.

33. Shroyer DA. Vertical maintenance of dengue-1 in sequential generations of Aedes albopictus. J Am Mosq Control Assoc. 1990;6:312-4.

34. Gratz NG. Critical review of vector status of Aedes albopictus. Med Vet Entomol. 2004;18:215-27.

35. Escobar J P, López Y L, Osorio L, González MC, Wolf MI. Plan de vigilancia para Aedes albopictus. En: Manual para la vigilancia y control de vectores de malaria, dengue, fiebre amarilla, leishmaniasis, enfermedad de Chagas y encefalitis equina venezolana desde el nivel municipal. Medellín: Dirección Seccional de Salud de Antioquia; 1999. p.86-92.

36. Darsie RF Jr. A taxonomic separation of Aedes albopictus from mosquitoes in the greater Antilles of the Caribbean area (Diptera, Culicidae). Mosq Syst. 1988;20:357-69. 